\title{
Ensuring Quality in Thyroid Cancer Surgery
}

\author{
Maria F Bates, Kristin L Long, and Rebecca S Sippel \\ Department of Surgery, University of Wisconsin, Madison, Wisconsin, US
}

DOI: https://doi.org/10.17925/USE.2017.13.01.22

$\mathrm{T}$ hyroid cancer incidence is increasing worldwide. Though long-term survival rates are excellent, recurrence remains a significant problem, which highlights potential areas of needed improvement, including the surgical care of these patients. This review paper identifies tools and markers that can be used to improve surgical quality in thyroid cancer. Preoperative surgical planning starts with an adequate ultrasound evaluation of the cervical lymph node basins. Postoperatively, thyroglobulin and radioactive iodine uptake scans can track adequacy of resection. In addition, lymph node yield and lymph node ratios serve as indirect markers for assessing the quality of lymph node dissections. Current research also suggests that high-volume surgeons have improved oncological outcomes. Surgeons can use these tools and information to follow and potentially improve the care provided to patients.

\section{Keywords}

Thyroid cancer, surgical quality, ultrasound, thyroglobulin, nodal dissection

Disclosure: Maria F Bates, Kristin L Long, and Rebecca S Sippel have nothing to declare in relation to this article. No funding was received in the publication of this article. This study involves a review of the literature and did not involve any studies with human or animal subjects performed by any of the authors.

Authorship: All named authors meet the International Committee of Medical Journal Editors (ICMJE) criteria for authorship of this manuscript, take responsibility for the integrity of the work as a whole, and have given final approval to the version to be published.

Open Access: This article is published under the Creative Commons Attribution Noncommercial License, which permits any noncommercial use, distribution, adaptation, and reproduction provided the original author(s) and source are given appropriate credit.

Received: February 21, 2017

Accepted: April 24, 2017

Citation: US Endocrinology, 2017;13(1):22-6

Corresponding Author: Maria F Bates, Department of Surgery, 600 Highland Avenue, K4/739 CSC, Madison, WI 53792, US. E: batesm@surgery.wisc.edu
Thyroid carcinoma is one of the most common solid tumors worldwide. The incidence of thyroid cancer is increasing, with an estimated 57,000 newly diagnosed cases in the US in 2017. Approximately three-quarters of these patients are female, with the majority being between 20 and 34 years of age. Though five-year survival rates are greater than $98 \%$ overall, the disease still inflicts significant morbidity on those affected. ${ }^{1}$ At present, surgery represents the mainstay for treatment and the only option for a definitive cure. Performing the appropriate operation for thyroid carcinoma is the first step towards achieving optimal outcomes. Current quality markers in thyroid surgery are predominately based on morbidity from often transient complications, such as temporary hypoparathyroidism or recurrent laryngeal nerve palsy. In order to improve the surgical care of patients with thyroid cancer, it is important that we focus not just on safety, but also on oncologic outcomes. Given the time delay to disease recurrence, surgeons are often not aware that disease recurrence has been identified or that the recurrence may have been due to inadequate initial surgical management. In a recent retrospective view of our institutional data, we collected all patients that underwent re-operative surgery for differentiated thyroid cancer. We found that the majority of patients had abnormal radiographic and/or elevated thyroglobulin ( $\mathrm{Tg}$ ) levels at their 6 month postoperative follow up from their initial surgery. Only three patients out of 92 actually had a negative ultrasound and undetectable $\mathrm{Tg}$ within 1 year, defining them as truly recurrent disease. By this definition, the remaining patients really just underwent a re-operation for persistent disease, which suggests a need for improved surgical quality. In order to improve the quality of thyroid cancer surgery, we need to identify short-term markers that can be used to give feedback to surgeons to allow them to improve their surgical care. In this review, we focus on the importance on quality surgery for thyroid cancer, specifically on the initial pre-operative evaluation, surgeon volume and outcomes, adequacy of surgical resection, and nodal disease. It is our hope that surgeons who perform surgery for thyroid cancer will use this information not only to track their own clinical outcomes but also to use these tools to re-evaluate the quality of care given to their patients.

\section{Quality surgery starts with a good pre-operative evaluation}

In order to achieve curative surgery as well as optimizing the effects of postoperative treatment and facilitate follow-up, surgeons must eradicate all the disease from the neck at the initial operation. Pre-operative radiographic imaging is the first step towards optimizing these outcomes. The neck ultrasound has emerged as the recommended first-line imaging modality to evaluate, not only disease within the thyroid gland, but also any potential spread to the central or lateral nodal basins in the neck. The most recent American Thyroid Association (ATA) guidelines recommend a formal pre-operative neck ultrasound evaluation of nodal disease prior to undergoing any thyroid surgery., ${ }^{2,3}$ This is further supported by Kocharyan and colleagues who demonstrated that lymph node disease identified on pre-operative ultrasound accurately corresponds to postoperative lymph node pathology. ${ }^{4}$ 
Pre-operative ultrasound evaluation primarily determines the extent of surgery performed and assists in determining prognosis. The quality of ultrasound exams of the neck is very operator-dependent and there is significant variability in interpretation of this modality. One recent study focused on the implementation of pre-operative ultrasound evaluation of lymph node disease in patients with well-differentiated thyroid carcinoma. This study demonstrated that high-quality cervical ultrasound changed the operative approach in $23 \%$ of patients, where a majority underwent more extensive operations for otherwise clinically occult lymph node metastases. ${ }^{5}$ Unfortunately, not all ultrasounds are equal and significant variability exists between non-specialist performed neck ultrasounds and specialist-performed neck ultrasounds. Mazzaglia evaluated 261 patients treated by a single surgeon for management of their thyroid disease over a two-year period, all of whom had an outside neck ultrasound available for comparison. Surgeon performed ultrasound (SPUS) was conducted on all patients by one surgeon, and $18 \%$ of the cohort had a change in treatment plans due to ultrasound discrepancies between the outside ultrasound and the SPUS. In eight of 132 patients undergoing thyroidectomy, the operative plan was significantly altered based on the results of the SPUS. ${ }^{6}$ Similar studies have also noted the superiority of the surgeon-performed ultrasound?

At our institution, we compared outcomes for thyroid cancer patients with pre-operative ultrasounds performed by a thyroid specialist versus a non-specialist. We demonstrated that a thyroid specialist was much more likely to document lymph status (69 versus 20\%). ${ }^{8}$ In addition, patients with a pre-operative ultrasound performed by a specialist had lower uptake on postoperative radioactive iodine scans and lower recurrence rates compared to those who had an ultrasound performed by a nonspecialist. In those patients with a pre-operative ultrasound performed by a non-specialist, a significant number of recurrences arose less than 12 months after their initial surgery. These early recurrences are likely secondary to inadequate initial surgery due to poor pre-operative recognition of the extent of disease. ${ }^{8}$ These findings prompted radiologists to re-evaluate their techniques and create a standardized approach to neck ultrasound for thyroid cancer that incorporates the ATA guidelines as well as focuses specific attention to the pertinent questions for endocrine surgeons.? In summary, any patient undergoing thyroid surgery should have a thorough evaluation of the cervical lymph node basins prior to surgery. In addition, it is important to consider the experience of the proceduralist performing the ultrasound and his or her familiarity with cervical neck ultrasound for thyroid carcinoma.

Abnormal lymph node features on ultrasound include larger size, loss of fatty hilum, round rather than oval shape, hyper-echogenicity, cystic changes, micro-calcifications, and peripheral vascularity. Of these variables, studies have demonstrated that peripheral vascularity and micro-calcifications show the highest sensitivity and specificity for detecting metastatic disease. ${ }^{10,11}$ Once identified by ultrasound or physical exam, abnormal lymph nodes in the lateral neck should undergo cytological confirmation with fine needle aspiration (FNA) in order to determine the extent of dissection needed. When cytology is inadequate or ultrasound and cytology findings are discordant, $\mathrm{Tg}$ washout testing can be done to confirm the presence of metastatic disease. ${ }^{12,13}$ Pre-operative FNA is not always necessary to evaluate central neck lymph nodes. These nodes can be difficult to access with FNA when the thyroid is in situ and can be easily assessed during the thyroid operation. It is best to perform a central lymph node dissection concomitant to thyroid surgery as the procedures utilize the same incision. Therefore, if there are concerning findings on pre-operative ultrasound within the central neck, a central neck dissection during the initial operation best serves the patient.

If cytology of a lymph node is positive for malignancy or Tg washout levels return elevated, then a compartment-oriented neck dissection should be performed. This should specifically include level VI (central neck, bounded by carotid arteries laterally, hyoid bone superiorly, and sternal notch inferiorly) for positive level VI nodes and a lateral neck dissection (levels II-V) for positive lateral neck nodes. ${ }^{14}$ In the lateral neck, all levels should be evaluated, as upper pole lesions will often present with disease in level II (upper jugular vein group) and may skip level VI, which is commonly considered the first site of metastasis. ${ }^{15}$ Wu et al. examined patients with papillary thyroid cancer (PTC) that underwent lateral neck dissection and demonstrated that patients with lateral neck nodal disease tend to have multiple involved nodes within multiple neck levels, where pre-operative ultrasound may not account for all involved nodes. Therefore, the surgeon should thoroughly dissect nodes in levels Ila-V, and he or she should avoid "berry picking" or node sampling in order to minimize risk of persistent disease. A compartment-oriented dissection should always be performed and a minimum of levels III and IV should be removed in all patients. Removal of levels Vb and Ila may not always be required, but should be pursued if there are any concerning findings on imaging or there is significant nodal burden in levels III and IV. Metastatic disease to level I nodes is infrequent and therefore is traditionally not included. ${ }^{16}$

\section{High-volume surgeons have better outcomes}

Current trends in many areas of surgery are heading towards subspecialization and this is also the case with thyroid cancer surgery. Subspecialization improves patient outcomes, decreases complications, increases efficiency, shortens hospital stays, increases patient satisfaction, and improves cost savings. These benefits apply similarly when comparing high-volume thyroid surgeons to low-volume thyroid surgeons. Recent studies demonstrate lower complication rates (hypocalcemia, nerve injuries, bleeding, and surgical site infections) as well as shorter lengths of stay for surgeons performing a high volume of thyroid operations in comparison to low volume surgeons. ${ }^{17-20}$ Adam and colleagues looked at almost 17,000 patients from the National Inpatient Sample and determined that a surgeon volume threshold of $>25$ total thyroidectomies per year was associated with decreased complications and shorter hospital stays. ${ }^{21}$ Another group compared financial costs of high versus low volume surgeons considering both cost of complications and cost of hospital stay. Low-volume surgeons cost approximately $\$ 10,000$ per patient compared to $\$ 6,662$ for high volume surgeons, estimating a cost saving of 3 billion dollars over 14 years if patients were referred only to high-volume surgeons. ${ }^{22}$

While cost and safety are important considerations, it is also important to examine the quality of patients' oncologic outcomes to determine if surgical volume improved patient outcomes when focusing on thyroid cancer recurrence and persistence. Adkisson and colleagues evaluated patients undergoing thyroidectomy over a one-year period and grouped surgeons by the number of thyroidectomies performed in that one year. They used radioactive iodine (RAl) uptake pre-scan, Tg levels, and RAl dose administered as markers of complete resection. Surgeons that performed $>30$ thyroidectomies a year were more likely to perform a more complete resection compared to those with less than 30 operations. For 
patients with advanced disease, $>50$ thyroidectomies a year were needed before improvements were seen in RAl uptake. This study represents an indirect measurement of surgical quality. ${ }^{23}$ Based on these results, high-volume thyroid cancer surgeons should be referred the patients with more extensive disease.

\section{Assessing the adequacy of surgical resection}

Measuring and determining quality in surgery is far from straightforward or simple. Early reports of quality metrics focused primarily on postoperative complications such as hypocalcemia, recurrent laryngeal nerve injury, bleeding, and surgical site infection. However, those metrics are not cancer-specific and do not reflect the adequacy nor appropriateness of the surgical resection. When discussing quality in thyroid cancer surgery, it is important to focus on oncologic outcomes, specifically recurrence and mortality. Mortality from well-differentiated thyroid carcinoma is very rare and true recurrences can take years or decades, making short-term markers of surgical quality incredibly important. Completeness of the initial thyroidectomy, appropriate pre-operative evaluation of lymph nodes, and adequacy of Iymph node dissection are appropriate and useful markers of surgical quality.

It has been shown that extent of resection influences both recurrence and mortality in differentiated thyroid cancer. ${ }^{24}$ Unfortunately, the amount of tissue left behind varies from surgeon to surgeon, even in operations labeled "total thyroidectomy." RAl scans are one method for evaluating the extent of resection. Traditionally, patients receive RAl a few months after surgery, and then they undergo a whole body scan 3-7 days later. From this scan, a percent remnant uptake is determined, which is the percent of dose detected in the thyroid bed. This is an indirect quantitative assessment of how much thyroid tissue remains (both normal and cancerous). We previously studied patients with differentiated thyroid cancer (DTC) who underwent total thyroidectomy and then received RA postoperatively. ${ }^{25}$ Eight surgeons were included and were classified as high volume if they performed $>20$ thyroidectomies per year. We calculated an uptake to dose ratio (UDR) for each patient, which demonstrated that UDR was 10-fold higher in patients who later presented with a recurrence. Therefore, we could use the percent uptake on a postoperative ablation scan to identify patients at "high risk" for recurrence. We could also use that uptake to identify patients that may not have had as "complete" an operation. We also examined the percent uptake related to surgical volume and we found that high-volume surgeons had a statistically significant lower UDR compared to low-volume surgeons, which means that they were removing more thyroid tissue and/or leaving behind less nodal disease. Interestingly, despite the likely more extensive surgical resection by the high-volume surgeons, they also had lower rates of complications, suggesting that a high-volume surgeon can safely perform surgery that is more extensive. ${ }^{25}$ This finding suggests that remnant uptake predicts recurrence and assists in judging the quality of the initial surgical management. These results provide surgeons with valuable feedback that may help them to improve their surgical outcomes.

Not all patients receive RAl, and therefore do not undergo uptake scans; therefore, this marker cannot be used in all patients. In addition, it would be ideal if we could identify those patients with likely residual disease prior to treating them with RAl, which will likely be ineffective at treating gross residual disease. Surgeons and endocrinologists utilize thyroglobulin as a tumor marker for differentiated thyroid carcinoma.
While typically not obtained until six months after surgery, more recently it has been found that the Tg levels nadir by $4-6$ weeks after surgery and that checking a level at that time can be used to guide postoperative treatment, specifically the need for radioactive iodine ablation or the need for repeat surgical intervention. ${ }^{26}$ Several studies show that undetectable postoperative Tg levels correspond with a low incidence of recurrence, while elevated Tg levels are associated with recurrence..$^{27,28}$ Rosario and colleagues demonstrated that Tg levels often detected recurrent papillary thyroid carcinoma, even prior to structurally detectable disease on ultrasound. ${ }^{29}$ One study evaluating the risk of recurrent PTC demonstrated an increase in $\mathrm{Tg}$ more than twofold was seen in $80 \%$ of patients with recurrence and only $13 \%$ of patients without demonstrable recurrent PTC on imaging. ${ }^{30}$

Tg can also be used to assess the adequacy of surgical management and to guide postoperative therapy. At our institution, we routinely obtain a six-week postoperative unstimulated Tg level and TSH prior to any RAI treatment. If the $\mathrm{Tg}<0.2 \mathrm{ng} / \mathrm{mL}$ most patients forego RAl; if the $\mathrm{Tg}<2 \mathrm{ng} / \mathrm{mL}$ RAl is considered; if $\mathrm{Tg}>2 \mathrm{ng} / \mathrm{mL}$ further imaging is obtained to identify disease that was presumably left behind. If surgeons identify structural disease on imaging, we refer these patients for re-resection. In a recent study, one group used thyroglobulin and thyroglobulin antibodies (TgAb) to compare the quality of surgery for thyroid cancer between open thyroidectomy and robotic thyroidectomy. Tg and TgAb levels were similar between the two groups at five years, and there were no differences in disease-free survival. ${ }^{31}$ As this group used Tg as a marker of surgical quality, a similar approach could be established among thyroid cancer surgeons. Surgeons should routinely document Tg levels postoperatively and review institutional outcomes to help drive towards continued improvement in both pre-operative and operative treatment of thyroid cancer patients.

\section{Assessing the quality of a nodal dissection}

In addition to completeness of thyroidectomy, the surgical community also needs tools to evaluate the adequacy of a lymph node dissection. The extent of dissection for metastatic lymph node disease varies greatly among surgeons. No metrics currently exist to evaluate how the extent of lymph node metastases influences oncologic outcomes. For example, at our clinic, we currently have a 46-year-old female who recently underwent total thyroidectomy and bilateral central neck dissection for PTC with pathology reporting a $3.2 \mathrm{~cm}$ PTC and 11 of 12 central neck nodes were positive for metastatic disease. Based on this pathology, how does one determine the adequacy of the lymph node dissection? In addition, how can we use this information on nodal involvement to determine her risk of recurrence?

Schneider et al. reviewed the surveillance, epidemiology, and end results (SEER) database to determine if lymph node ratio (LNR) (number of metastatic nodes/number of nodes removed) could predict the risk of mortality in patients with PTC who underwent thyroidectomy and lymph node dissection. Using Kaplan-Meier survival estimates, they demonstrated that patients with a LNR $\geq 0.42$ experienced a disease specific mortality rate of $1.72 \%$ while those $<0.42$ had a rate of $0.65 \%{ }^{32}$ since mortality is rare in PTC, disease recurrence is a more relevant oncologic outcome to examine. We further conducted a retrospective cohort review of patients undergoing total thyroidectomy with lymph node dissection and sought to determine if LNR could predict disease recurrence. When trying to risk stratify variables as predictors of recurrence, we performed a multivariate analysis looking at classic high-risk variables such as multi-focality, extra- 
thyroidal extension, lympho-vascular invasion, positive margins, etc. When LNR ratio was included in the model, it clearly dominated every other factor and was by far the strongest predictor of recurrence. Thus, we concluded that elevated LNR was significantly associated with disease recurrence and is probably the strongest predictor of disease recurrence after surgery. ${ }^{33}$ Lee and colleagues went further and studied a cohort of almost 2,300 patients with PTC who had undergone total thyroidectomy. Six percent of patients presented with recurrence with a median follow-up period of 107 months. Using prediction probability, they determined LNR ratio cutoff of 0.4 and 0.5 for central and lateral neck dissections, respectively. Recurrence-free survival was analyzed using the above cutoff points, which demonstrated that $L N$ ratio $>0.4$ to central neck and $>0.5$ for lateral neck compartments corresponded to significantly decreased rates of recurrence free survival. ${ }^{34}$

Lymph node yield (LNY) and lymph node size may also have implications for recurrence. Heaton and colleagues evaluated lymph node yield from central and lateral neck dissections of patients with papillary thyroid carcinoma. They found that patients with recurrence had an average LNY in the central neck of 2.5 nodes, while those without recurrence had an average LNY of 10.3 nodes. Similar findings were observed with lateral neck dissections with node averages of 10.5 versus 24.6 for those with recurrence and those without, respectively. ${ }^{35}$ These findings suggest that LNY may also be used as a marker of surgical adequacy, however, an earlier study did not find any correlation with LNY, but did correlate higher lymph node ratios with increased recurrence. ${ }^{36}$ In addition, the size of the largest metastatic lymph also upstages the recurrence risk. ${ }^{37}$ In the most recent ATA 2015 guidelines for well-differentiated thyroid cancer, lymph node size $>3 \mathrm{~cm}$ is consider a high-risk feature, suggesting that size also matters here. ${ }^{2}$

The above studies demonstrate that LNY, largest involved lymph node, and especially LNR are valuable tools in the management and treatment of DTC. The key to performing a lymph node dissection well is to remove not just the immediate extent of disease but also the rim of normal lymph nodes beyond the obvious extent of disease to ensure removal of any adjacent microscopic disease. By aiming to achieve a LNR of about 0.5 when performing a lymph node dissection, you can significantly reduce the risk of recurrence and improve long-term outcomes for patients. LNR also helps determine risk of recurrence and if there is need for additional surgery. If patients have a high LNR and, consequently a high risk of recurrence, surgeons can tailor their postoperative treatment and followup plan to consider this finding. By tracking this outcome, surgeons can also use this as a marker to help assess the adequacy of their pre-operative nodal evaluation and the extent of their lymph node dissection. Our goal as surgeons should not just be to remove the obvious disease, but also to strive to do what we can to minimize the risk of the patient needing an additional operation in the future.

\section{Conclusion}

Surgical therapy for thyroid carcinoma has evolved over time and continues to change each year as more research emerges in this area. Here, we focused on quality in thyroid cancer surgery. When evaluating quality in thyroid cancer surgery, one should not only concentrate on complications, but one should also place more weight on improving long-term outcomes with specific focus on disease recurrence. Again, we emphasize that surgical treatment effectively begins in the pre-operative period with accurate quantification of the burden of disease facilitated by quality radiographic imaging. It is crucial that all patients undergoing thyroid surgery receive a comprehensive cervical neck ultrasound including lymph node assessment of all compartments of the neck. This key step is crucial in determining what the most appropriate initial operation is and is paramount to optimizing long-term oncological outcomes.

In addition, we discussed radioactive iodine remnant uptake, postoperative thyroglobulin levels, and lymph node ratios as tools to help guide quality care in thyroid surgery. Surgeons can use these tools in everyday practice. For example, at our clinic we currently have a 45-year-old female and her immediate postoperative $\mathrm{Tg}$ level is elevated. This reflects the presence of remaining thyroid tissue or thyroid cancer. Using this information, the surgeon can re-evaluate the adequacy and quality of the pre-operative ultrasound and re-evaluate the adequacy and quality of the surgery itself. Furthermore, with an elevated postoperative Tg, depending on the level, patients may need more radiographic evaluation looking for persistent disease left behind and/or RAl for ablation of the remaining tissue. In addition, when performing lymph node dissections, surgeons should aim to adequately clear the disease and aim for lower lymph node ratios. Not only does this reflect the adequacy of the surgery and can be used to guide surgical improvement, but the LNR can also be used to risk stratify the patient for recurrence and to guide postoperative treatments and follow up.

It is important that surgeons continue to strive for improvement in order to perform the best oncological operation for the patient. If we can improve the surgical care of thyroid cancer, we can minimize the need for additional therapy, and optimize long-term outcomes for our patients. $]$
1. American Cancer Society, Cancer Facts \& Figures, Atlanta: American Cancer Society, 2017.

2. Haugen BR, Alexander EK, Bible KC, et al., 2015 American Thyroid Association Management Guidelines for Adult Patients with Thyroid Nodules and Differentiated Thyroid Cancer: The American Thyroid Association Guidelines Task Force on Thyroid Nodules and Differentiated Thyroid Cancer, Thyroid, 2016;26:1-133.

3. Yeh MW, Bauer AJ, Bernet VA, et al., American Thyroid Association statement on preoperative imaging for thyroid cancer surgery, Thyroid, 2015:25:3-14.

4. Kocharyan D, Schwenter F, Belair M, Nassif E, The relevance of preoperative ultrasound cervical mapping in patients with thyroid cancer, Can J Surg, 2016;59:113-7.

5. O'Connell K, Yen TW, Quiroz F, et al., The utility of routine preoperative cervical ultrasonography in patients undergoing thyroidectomy for differentiated thyroid cancer, Surgery, 2013;154:697-701; discussion -3.

6. Mazzaglia PJ, Surgeon-performed ultrasound in patients referred for thyroid disease improves patient care by minimizing performance of unnecessary procedures and optimizing surgical treatment, World J Surg, 2010;34:1164-70.

7. Carneiro-Pla D, Amin S, Comparison between preconsultation ultrasonography and office surgeon-performed ultrasound in patients with thyroid cancer, World I Surg, 2014:38:622-7.

8. Oltmann SC, Schneider DF, Chen H, Sippel RS, All thyroid ultrasound evaluations are not equal: sonographers specialized in thyroid cancer correctly label clinical $\mathrm{NO}$ disease in well differentiated thyroid cancer, Ann Surg Oncol, 2015;22:422-8.

9. Kumbhar SS, O'Malley RB, Robinson TJ, et al., Why Thyroid Surgeons Are Frustrated with Radiologists: Lessons Learned from Pre- and Postoperative US, Radiographics, 2016;36:2141-53.

10. LeboulleuX S, Girard E, Rose M, et al., Ultrasound criteria of malignancy for cervical lymph nodes in patients followed up for differentiated thyroid cancer, I Clin Endocrinol Metab, 2007;92:3590-4.

11. Liu Z, Zeng W, Liu C, et al., Diagnostic accuracy of ultrasonographic features for lymph node metastasis in papillary thyroid microcarcinoma: a single-center retrospective study, World J surg Oncol, 2017:15:32.

12. Snozek $C L$, Chambers EP, Reading $C C$, et al., Serum thyroglobulin, high-resolution ultrasound, and lymph node thyroglobulin in diagnosis of differentiated thyroid carcinoma nodal metastases, J Clin Endocrinol Metab, 2007;92:4278-81.
13. Grani G, Fumarola A, Thyroglobulin in lymph node fine-needle aspiration washout: a systematic review and meta-analysis of diagnostic accuracy, J Clin Endocrinol Metab, 2014:99:1970-82.

14. Al-Hilli Z, Strajina V, MCKenzie TJ, et al., The role of lateral neck ultrasound in detecting single or multiple lymph nodes in papillary thyroid cancer, Am J Surg, 2016;212:1147-53.

15. Park JH, Lee YS, Kim BW, et al., Skip lateral neck node metastases in papillary thyroid carcinoma, World I Surg, 2012;36:743-7.

16. Wu G, Fraser S, Pai SI, et al., Determining the extent of lateral neck dissection necessary to establish regional disease contro and avoid reoperation after previous total thyroidectomy and radioactive iodine for papillary thyroid cancer, Head Neck, 2012;34:1418-21.

17. Kandil E, Noureldine SI, Abbas A, Tufano RP, The impact of surgical volume on patient outcomes following thyroid surgery, Surgery, 2013;154:1346-52; discussion 52-3.

18. Meltzer C, Klau M, Gurushanthaiah D, et al., Surgeon volume in thyroid surgery: Surgical efficiency, outcomes, and utilization, thyroid surgery: Surgical efficiency, outco

19. Nouraei SA, Virk JS, Middleton SE, et al., A national analysis of trends, outcomes and volume-outcome relationships in thyroid surgery, Clin Otolaryngol, 2017;42:354-65 
20. Sosa JA, Bowman HM, Tielsch JM, et al., The importance of surgeon experience for clinical and economic outcomes from thyroidectomy, Ann Surg, 1998;228:320-30

21. Adam MA, Thomas $S$, Youngwirth $L$, et al., Is there a mnimum number of thyroidectomies a surgeon should perform to optimize patient outcomes?, Ann Surg, 2017:265:402-7.

22. Al-Qurayshi Z, Robins R, Hauch A, et al., Association of surgeon volume with outcomes and cost savings following thyroidectomy: A national forecast, JAMA Otolaryngol Head Neck Surg, 2016;142:32-9.

23. Adkisson CD, Howell GM, McCoy KL, et al., Surgeon volume and adequacy of thyroidectomy for differentiated thyroid cancer, Surgery, 2014;156:1453-59; discussion 60.

24. Bilimoria $\mathrm{KY}$, Bentrem DJ, Ko CY, et al., Extent of surgery affects survival for papillary thyroid cancer, Ann Surg, 2007;246:375-81; discussion 81-4

25. Schneider DF, Ojomo KA, Chen $H$, Sippel RS, Remnant uptake as a postoperative oncologic quality indicator, Thyroid, 2013;23:1269-76.

26. Kashat L, Orlov S, Orlov D, et al., Serial post-surgical stimulated and unstimulated highly sensitive thyroglobulin measurements in low- and intermediate-risk papillary thyroid carcinoma patients not receiving radioactive iodine, Endocrine, 2016;54:460-6. 27. Janovsky CC, Maciel RM, Camacho CP, et al., A prospective study showing an excellent response of patients with low-risk differentiated thyroid cancer who did not undergo radioiodine remnant ablation after total thyroidectomy, Eur Thyroid J,

2016:5:44-9.

28. Lee HS, Roh JL, Gong G, et al., Risk factors for re-recurrence after first reoperative surgery for locoregional recurrent/persistent papillary thyroid carcinoma, World I Surg, 2015;39:1943-50

29. Rosario PW, Mourao GF, Calsolari MR, Can the follow-up of patients with papillary thyroid carcinoma of low and intermediate risk and excellent response to initial therapy be simplified using second-generation thyroglobulin assays? Clin Endocrinol (Oxf). 2016;85:596-601.

30. Choudhary C, Wartofsky L, Tefera E, Burman KD, Evaluation of thyroid bed nodules on ultrasonography after total thyroidectomy: Risk for loco-regional recurrence of thyroid cancer, Eur Thyroid J, 2015;4:106-14

31. Lee SG, Lee J, Kim MJ, et al., Long-term oncologic outcome of robotic versus open total thyroidectomy in PTC: a case-matched retrospective study, Surg EndosC, 2016;30:3474-9.
32. Schneider DF, Chen H, Sippel RS, Impact of lymph node ratio on survival in papillary thyroid cancer, Ann Surg Oncol, 2013;20:1906-11.

33. Schneider DF, Mazeh $\mathrm{H}$, Chen $\mathrm{H}$, Sippel RS, Lymph node ratio predicts recurrence in papillary thyroid cancer, The Oncologist, 2013; 18:157-62.

34. Lee SG, Ho J, Choi JB, et al., Optimal cut-off values of lymph node ratio predicting recurrence in papillary thyroid cancer, Medicine 2016;95:e2692.

35. Heaton CM, Chang JL, Orloff LA, Prognostic implications of lymph node yield in central and lateral neck dissections for well-differentiated papillary thyroid carcinoma, Thyroid 2016;26:434-40

36. Vas Nunes JH, Clark JR, Gao K, et al., Prognostic implications of lymph node yield and lymph node ratio in papillary thyroid carcinoma, Thyroid, 2013;23:811-6.

37. Randolph GW, Duh QY, Heller KS, et al., The prognostic significance of nodal metastases from papillary thyroid carcinoma can be stratified based on the size and number of metastatic lymph nodes, as well as the presence of extranodal extension, Thyroid, 2012;22:1144-52. 